\title{
Spatially Controlled Octahedral Rotations and Metal-Insulator Transitions in Nickelate Superlattices
}

\author{
Binbin Chen, Nicolas Gauquelin, Robert J. Green, Jin Hong Lee, Cinthia Piamonteze, Matjaž Spreitzer, \\ Daen Jannis, Johan Verbeeck, Manuel Bibes, Mark Huijben, Guus Rijnders, and Gertjan Koster*
}

Cite This: Nano Lett. 2021, 21, 1295-1302

Read Online

\section{ACCESS | Lلll Metrics \& More | 回 Article Recommendations | S1 Supporting Information}

ABSTRACT: The properties of correlated oxides can be manipulated by forming short-period superlattices since the layer thicknesses are comparable with the typical length scales of the involved correlations and interface effects. Herein, we studied the metal-insulator transitions (MITs) in tetragonal $\mathrm{NdNiO}_{3} /$ $\mathrm{SrTiO}_{3}$ superlattices by controlling the $\mathrm{NdNiO}_{3}$ layer thickness, $n$ in the unit cell, spanning the length scale of the interfacial octahedral coupling. Scanning transmission electron microscopy reveals a crossover from a modulated octahedral superstructure at $n=8$ to a uniform nontilt pattern at $n=4$, accompanied by a drastically weakened insulating ground state. Upon further reducing $n$ the predominant dimensionality effect continuously raises the MIT
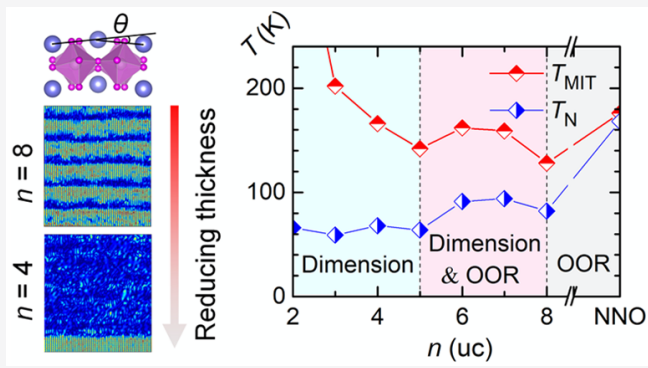
temperature, while leaving the antiferromagnetic transition temperature unaltered down to $n=2$. Remarkably, the MIT can be enhanced by imposing a sufficiently large strain even with strongly suppressed octahedral rotations. Our results demonstrate the relevance for the control of oxide functionalities at reduced dimensions.

KEYWORDS: metal-insulator transition, oxygen octahedral rotations, dimensionality, strain, nickelate superlattice

$\mathrm{T}$ he ubiquitous electron-lattice coupling in perovskite oxides enables us to tune their functionalities by virtue of structural engineering. ${ }^{1-10}$ In addition to the well-established strain approach, engineering of the oxygen octahedral rotations (OOR) has been recognized as another effective tool since both the electronic bandwidth and magnetic exchange interaction in oxides are sensitive to the changes of the $\mathrm{M}-$ $\mathrm{O}-\mathrm{M}$ ( $\mathrm{M}$, transition metal ion) bond angle. ${ }^{3-10}$ At the interface between two perovskites, the corner-shared octahedral network has to rearrange to maintain the connectivity, known as the octahedral proximity effect. ${ }^{3}$ Such an effect is usually confined within several unit cells (uc) and rapidly decays away from the interface, ${ }^{3-10}$ in contrast to the longrange effect up to tens of nanometers for strain engineering. ${ }^{1,2}$ By forming short-period superlattices (SLs), the interfacial OOR coupling can be extended throughout the entire film, thus creating novel structural phases with distinct properties from the individual components. ${ }^{7,11,12}$ Importantly, the OOR can be manipulated in SLs by designing the relative thickness ratio of the component layers or selectively utilizing a tiltcontrol layer, offering extra tuning "knobs"., when the layer thickness is reduced down to a few uc in SLs, a dimensional effect comes into play and usually reduces the bandwidth. ${ }^{13-19}$ The competition between the OOR and dimensional effects remains rarely explored ${ }^{20,21}$ but is crucial for the rational control over oxide functionalities at the atomic scale.
Rare-earth nickelates $\mathrm{RNiO}_{3}$ have received continuous attention not only because of their puzzling physics but also for the potential to design "Mottronics" devices. ${ }^{22,23}$ Except for $\mathrm{R}=\mathrm{La}, \mathrm{RNiO}_{3}$ displays a sharp metal-insulator transition (MIT) which can be systematically tuned by changing rareearth cations. Concurrent with the MIT, a structural transition from orthorhombic $(P b n m)$ to monoclinic $\left(P 2_{1} / c\right)$ symmetry occurs due to the breathing distortions of the $\mathrm{NiO}_{6}$ octahedra. This MIT is accompanied by a paramagnetic to antiferromagnetic transition for $\mathrm{R}=\mathrm{Pr}$ and $\mathrm{Nd}$, although the transitions are well separated for the smaller rare-earth ions. The mechanism for this temperature-driven MIT has been a longstanding debate. ${ }^{24-30}$ Recently, resonant inelastic X-ray scattering spectroscopy revealed a negative charge transfer energy and oxygen ligand holes $(\underline{\mathrm{L}})$ in $\mathrm{NdNiO}_{3}(\mathrm{NNO}){ }^{31}$ supporting a theoretically predicted bond disproportionation scenario. ${ }^{25}$ The ground state is then described as alternatively arranged $\mathrm{Ni} 3 \mathrm{~d}^{8}$ and $\mathrm{Ni} 3 \mathrm{~d}^{8} \underline{\mathrm{L}}^{2}$ sites, which are associated with the breathing mode of the $\mathrm{NiO}_{6}$ octahedra. ${ }^{30}$ The two inequivalent $\mathrm{Ni}-\mathrm{O}$ bonds can also account for the peculiar antiferromagnetic order at low temperatures. ${ }^{22}$ More recently,

Received: October 4, 2020

Revised: January 13, 2021

Published: January 20, 2021 
(a)

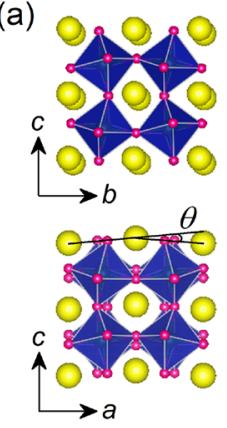

(b)

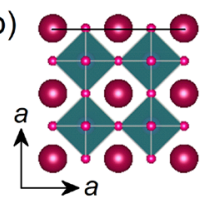

(c)

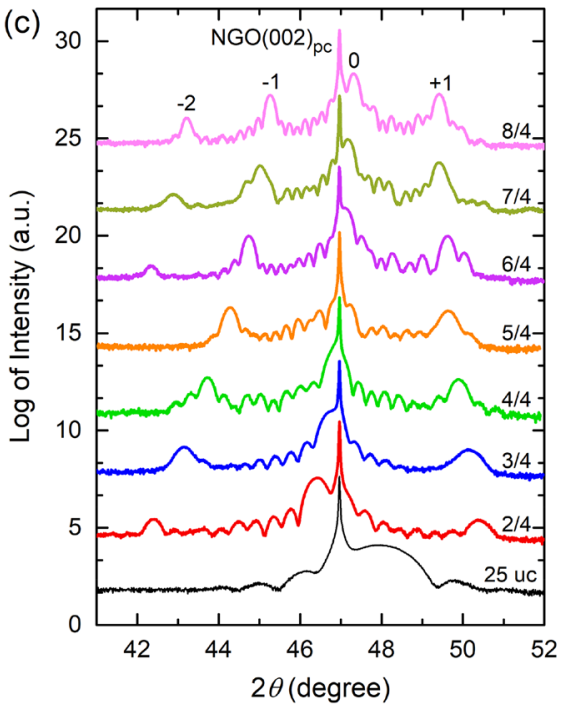

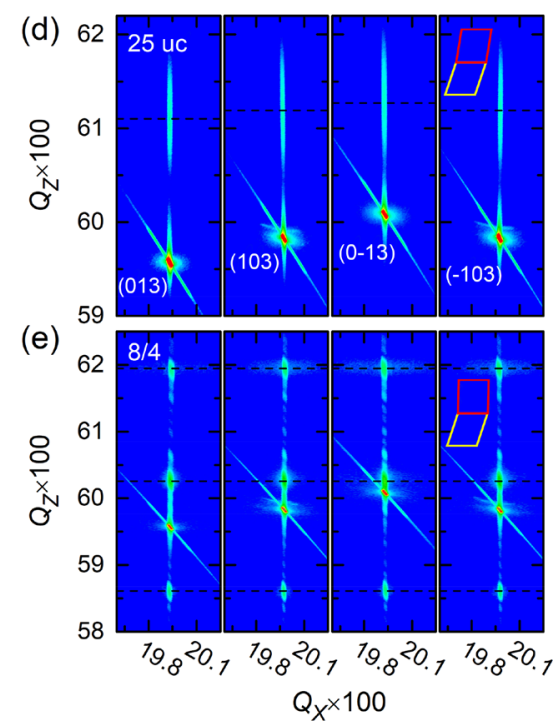

Figure 1. Structural characterization of NNO/STO SLs. Schematic lattice structure of NNO (a) and STO (b). The royal blue and dark cyan octahedra and yellow, wine, and magenta spheres represent $\mathrm{NiO}_{6}, \mathrm{TiO}_{6}, \mathrm{Nd}, \mathrm{Sr}$, and $\mathrm{O}$, respectively. (c) High-resolution XRD $\theta-2 \theta$ scans around the $\mathrm{NGO}(002)_{\mathrm{pc}}$ peak for the NNO/STO SLs with varied $n$. RSMs around pseudocubic (013), (103), (013), and (103) reflections measured from the 25-uc NNO single film (d) and the 8/4 SL (e). The schematic insets show the shear distortion of the pseudocubic lattice.
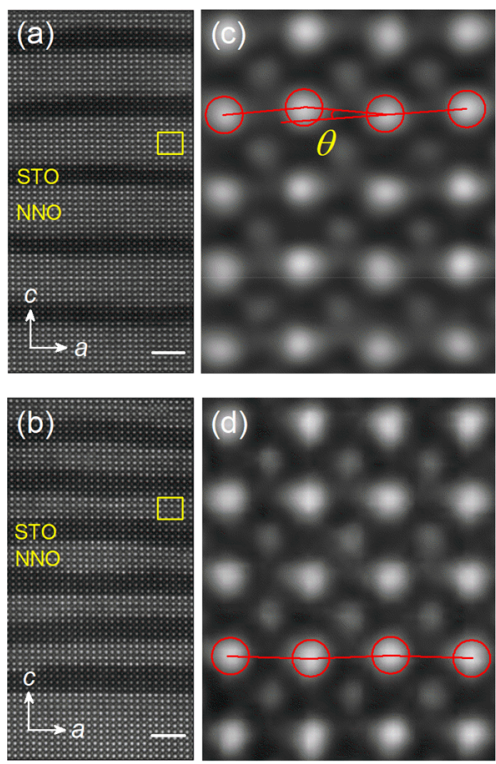

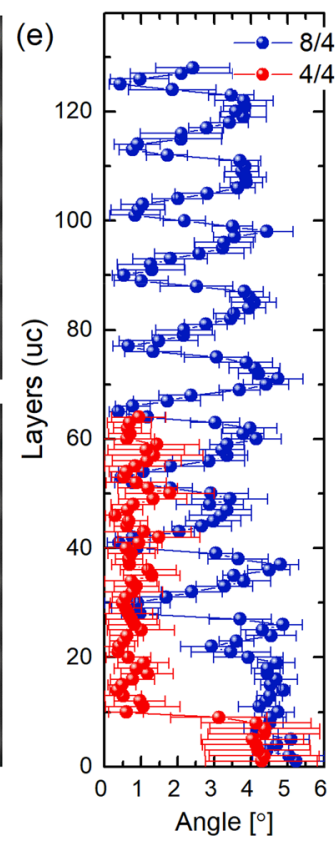

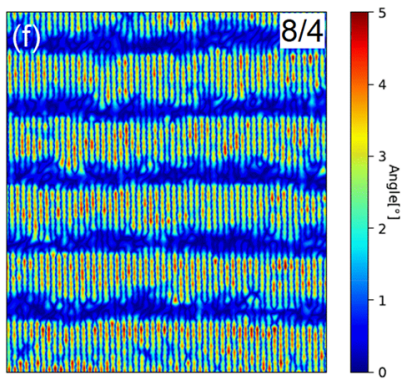

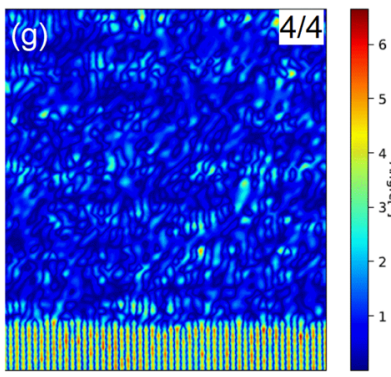

Figure 2. STEM characterization of OOR in NNO/STO SLs. STEM-HAADF images of NNO/STO SLs with $n=8$ (a) and $n=4$ (b). Scale bar, 2 $\mathrm{nm}$. The corresponding enlarged images are shown in $(\mathrm{c})$ and $(\mathrm{d})$ to highlight the A-site antipolar displacements. The deviation angle $\theta$ is defined as described in the main text. (e) Layer-resolved deviation angle $\theta$. Cross-sectional mapping of $\theta$ in the SLs with $n=8$ (f) and $n=4$ (g).

the intimate competition between the two main structural distortions in $\mathrm{RNiO}_{3}$, namely, the breathing mode and the OOR, has been unveiled by first-principles calculations, where a certain magnitude of OOR can trigger the breathing mode and induce the MIT. ${ }^{29}$

$\mathrm{RNiO}_{3}$ at reduced dimensions is of interest as a planar orbital order, similar to high-temperature superconducting cuprates, has been predicted. ${ }^{32,33}$ Aside from the orbital control, an emergent antiferromagnetic order, competing electronic phases, and enhanced charge-order propensity have been reported in artificially confined $\mathrm{RNiO}_{3}$ heterostructures. $^{13,15,34,35}$ Herein, the combined effects of the OOR and spatial confinement on the MIT were studied in NNO/ $\mathrm{SrTiO}_{3}$ (STO) SLs via digitally varying the NNO layer thickness, $n$ in the uc. We observed a nonmonotonic variation of the MIT temperature $\left(T_{\text {MIT }}\right)$ as a function of $n$ because of the competition between the OOR and dimensional crossover. On the other hand, the antiferromagnetic transition temperature $\left(T_{\mathrm{N}}\right)$ is found to be mainly controlled by the $\mathrm{Ni}-\mathrm{O}-\mathrm{Ni}$ bond angle and $\mathrm{Ni}-\mathrm{O}$ bond length but insensitive to the dimensional reduction. The distinct response of the MIT and the antiferromagnetic transition to dimensionality offers great opportunities to engineer novel phases in nickelate SLs. 

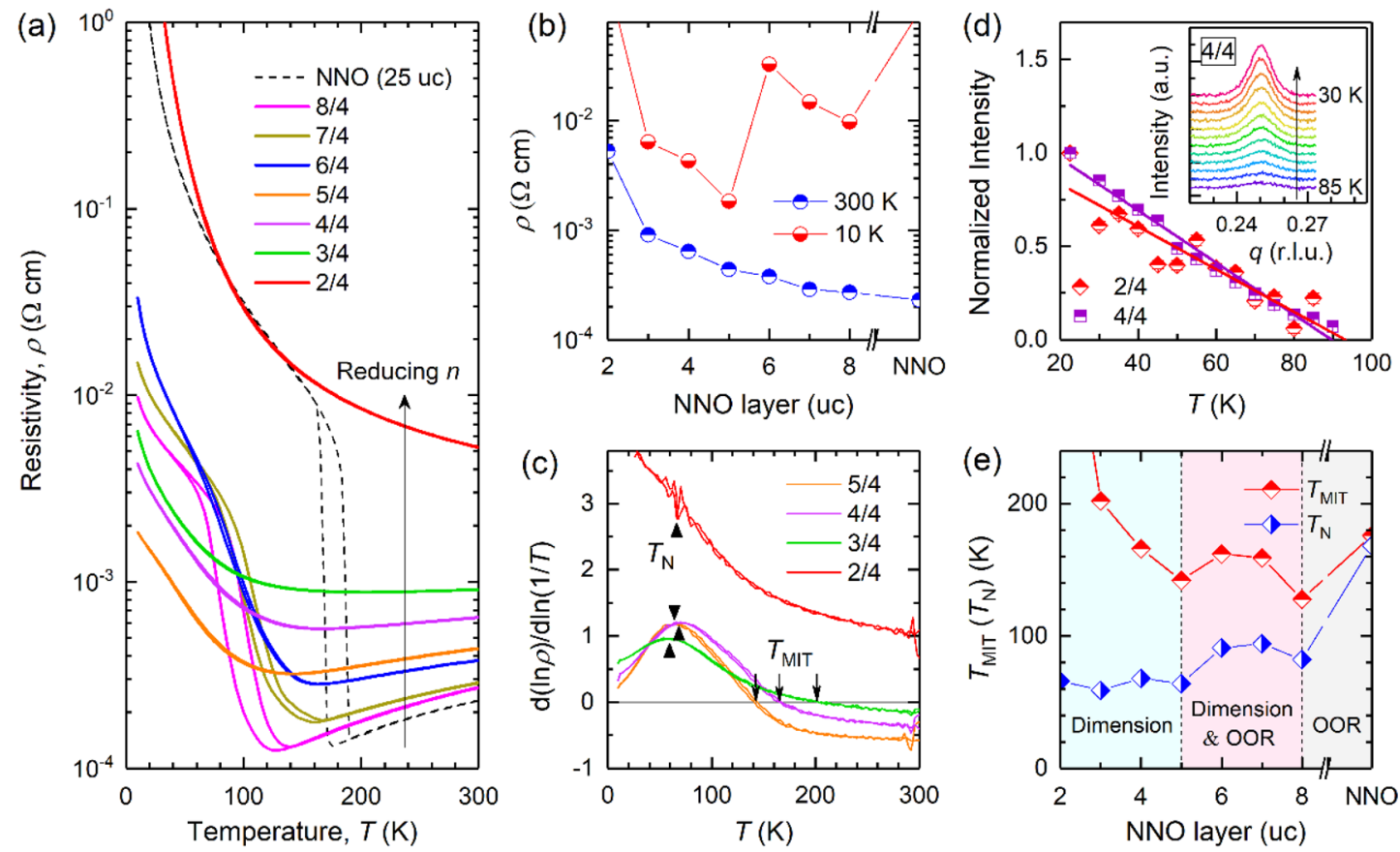

Figure 3. Thickness-dependent MITs in NNO/STO SLs. (a) Temperature dependent resistivity $(\rho-T)$ curves of NNO/STO SLs with varied $n$, along with a 25-uc-thick NNO single film for comparison. (b) Resistivities at 300 and $10 \mathrm{~K}$ as a function of $n$. (c) $\mathrm{d}(\ln \rho) /[\mathrm{d} \ln (1 / T)]$ vs $T$ plots for the SLs with $n=2,3,4$, and 5. $T_{\mathrm{MIT}}$ and $T_{\mathrm{N}}$, marked by the arrow and triangle, are defined as the temperature of the upturn in the $\rho-T$ curve and the peak in the $\mathrm{d}(\ln \rho) /[\mathrm{d} \ln (1 / T)]$ vs $T$ plot during the cooling process. (d) Temperature dependent integrated intensity of the $q=(1 / 4,1 / 4$, $1 / 4)_{p c}$ reflection measured for SLs with $n=2$ and 4 using RMD. The linear fitting is used to extract the onset temperature of the antiferromagnetic order. The inset shows the isothermal rocking curves around the $q=(1 / 4,1 / 4,1 / 4)_{\mathrm{pc}}$ reflection taken from the SL with $n=4$. The measurements were performed from 30 to $85 \mathrm{~K}$ with a step of $5 \mathrm{~K}$. (e) Phase diagram of NNO/STO SLs as a function of $n$. Here $T_{\mathrm{N}}$ is derived from the resistivity measurements.

The NNO/STO SLs, $n / 4$, were fabricated on $(001)_{\text {pc }^{-}}$ oriented (pc, pseudocubic) $\mathrm{NdGaO}_{3}(\mathrm{NGO})$ substrates by means of pulsed laser deposition monitored with reflection high-energy electron diffraction (RHEED) (Figure S1). We set the STO layer thickness to 4 uc and the repetition number of the NNO/STO bilayer to 10 . Bulk NNO is orthorhombic at room temperature showing an OOR pattern of $a^{+} b^{-} b^{-}$in Glazer notation (Figure 1a). Hereafter, we use the pseudocubic indices for NNO and NGO, with $a, b$, and $c$ corresponding to the $[001],[1 \overline{1} 0]$, and $[110]$ axes of the orthorhombic lattice. According to first-principles calculations, the combination of out-of-phase and in-phase rotations can drive antipolar displacements of the A-site cations to optimize the oxygen coordination, ${ }^{36}$ as visualized from the projection along the $b$ axis of NNO. In this work, we used the A-site displacements as a relative measure of the OOR considering the high accuracy in determining $\mathrm{Nd}^{3+}$ positions from scanning transmission electron microscopy (STEM). ${ }^{7,9,37}$ Unlike orthorhombic NNO, STO is cubic without any OOR and A-site displacements (Figure 1b). The symmetry mismatch at the heterointerface is expected to alter the OOR in the nearinterface region of NNO and tune its properties. ${ }^{3,5}$ Figure 1c shows the high-resolution $\theta-2 \theta$ X-ray diffraction (XRD) scans around the $\mathrm{NGO}(002)_{\mathrm{pc}}$ peak for SLs with varied $n$. The presence of satellite peaks and thickness fringes for all SLs attests to their abrupt interfaces and smooth surfaces. Atomic force microscopy further confirms the terraced surface (Figure S2). As $n$ reduces, the main peak gradually shifts to lower angles because of the increasing contribution from the compressively strained STO layer. Reciprocal space mappings (Figure 1d,e) reveal a change in crystal symmetry from orthorhombic in the NNO single film to tetragonal in the $8 / 4$ SL. An analogous structural change has also been reported in $\mathrm{SrRuO}_{3}$ films grown on $\mathrm{STO}$ when scaling down the thickness below a critical value. ${ }^{38}$ The tetragonal phase is ascribed to the suppressed OOR along the in-plane axes as evidenced by STEM and half-order XRD measurements. ${ }^{5,38,39}$ For the SLs with smaller $n$, the RSMs show the same features as the $8 / 4$ SL (Figure S3). Given the fact that XRD measurements provide spatially averaged structural information over the samples, we performed atomically resolved STEM measurements to reveal more delicate changes of the OOR within the NNO/STO SLs.

Figure 2a,b shows the high-angle annular dark field (HAADF)-STEM images of the $8 / 4$ and 4/4 SLs, respectively. Due to the large difference in atomic numbers between the $\mathrm{Sr}$ and the Nd atoms, the NNO and STO layers can be readily identified from the Z-contrast images as well as the electron energy loss spectroscopy (EELS) color maps (Figure S4). Figure $2 c, d$ shows the enlarged images of the NNO layer in the corresponding SLs to highlight the displacements of $\mathrm{Nd}^{3+}$. We define $\theta$ as the deviation of the angle between three successive $\mathrm{Nd}^{3+}$ cations along the $a$ axis from $180^{\circ}$. Note that $\theta$ scales with the OOR. ${ }^{7,9,37}$ We find that the $8 / 4$ SL shows pronounced antipolar displacements of $\mathrm{Nd}^{3+}$, which becomes negligible in the $4 / 4 \mathrm{SL}$. The quantified measure of $\theta$ is carried out along the growth direction as summarized in Figure 2e. A clear modulation of $\theta$ is observed in the 8/4 SL. The noninterfacial region of NNO shows a maximum $\theta$ of $\sim 4.0 \pm$ $0.5^{\circ}$ which decays in the vicinity of the interface. For the $4 / 4$ SL, we observe a uniform $\theta$ of $\sim 0.5^{\circ}$ throughout the SL. Therefore, the coherence length of the interfacial OOR coupling is around $\sim 2$ uc on the NNO side, which is 
comparable to those of the $\mathrm{SrIrO}_{3} / \mathrm{STO}$ and $\mathrm{La}_{0.7} \mathrm{Sr}_{0.3} \mathrm{MnO}_{3} /$ STO interfaces. ${ }^{21,40}$ Figure $2 \mathrm{f}, \mathrm{g}$ shows the cross-sectional mapping of $\theta$. The modulated $\theta$ in the $8 / 4 \mathrm{SL}$ is in sharp contrast with the nearly homogeneous distribution of $\theta$ in the 4/4 SL. The almost quenched A-site antipolar displacement indicates $a^{0} a^{0} c^{-}$for the $4 / 4 \mathrm{SL}^{41}$ This means the OOR along the in-plane axes is further quenched by reducing the NNO layer thickness.

The modifications to the structural ordering within the NNO/STO SLs are expected to cause significant variations to the transport properties. Figure $3 \mathrm{a}$ shows the temperature dependent resistivity curves of the NNO/STO SLs as well as a 25-uc thick NNO film for reference. The resistivities at $300 \mathrm{~K}$ $[\rho(300 \mathrm{~K})]$ and $10 \mathrm{~K}[\rho(10 \mathrm{~K})]$ are plotted against $n$ in Figure $3 \mathrm{~b}$. At first glance, as $n$ is reduced, $\rho(300 \mathrm{~K})$ gradually increases whereas $\rho(10 \mathrm{~K})$ shows a nonmonotonic variation. As we will discuss below, the monotonic increase of $\rho(300 \mathrm{~K})$ is related to the reduced dimensions as well as the increasing disorders, while the complex evolution of $\rho(10 \mathrm{~K})$ is due to the competition between the OOR and the dimensionality effect. The NNO film undergoes a sharp MIT as the temperature decreases below $176 \mathrm{~K}$, which is $24 \mathrm{~K}$ lower than the bulk value $(\sim 200 \mathrm{~K})$ because of the tensile strain. ${ }^{2,22,42}$ The resistivity shows a change of 4 orders of magnitude across the MIT, indicative of the high crystallinity and optimal oxygen stoichiometry of the film. ${ }^{42,43}$ In the $8 / 4$ SL $T_{\text {MIT }}$ drastically decreases to $128 \mathrm{~K}$. Also, the low-temperature insulating state is suppressed as manifested by the sharp decline of $\rho(10 \mathrm{~K})$ shown in Figure $3 \mathrm{~b}$. This is related to the suppression of orthorhombic distortions as evidenced by the RSM results in Figure 1d,e. The suppressed OOR along the in-plane axes stretches the $\mathrm{Ni}-\mathrm{O}-\mathrm{Ni}$ bond and boosts the bandwidth as revealed by X-ray absorption spectroscopy (XAS). ${ }^{9,10}$ Note that the transition hysteresis is similar to the NNO single film, which implies that the percolation behavior across the MIT is not interrupted in the $n=8 \mathrm{SL}^{22}$ However, the thermal hysteresis is markedly weakened upon reducing $n$ from 8 to 6 uc. Meanwhile, the $T_{\text {MIT }}$ gradually increases from 128 to $162 \mathrm{~K}$ accompanied by a rise of $\rho(10 \mathrm{~K})$. This is ascribed to the dimensionality effect which stabilizes the insulating phase by blocking the interlayer electron hopping. ${ }^{14-17}$ As a result, the region with coexisting metallic and insulating phases gradually vanishes. ${ }^{17}$ Most strikingly, as $n$ is further reduced, we observe an unexpected decline of $T_{\mathrm{MIT}}$ from $162 \mathrm{~K}$ at 6 uc to $142 \mathrm{~K}$ at $5 \mathrm{uc}$, concurrent with a sudden drop of $\rho(10 \mathrm{~K})$. The result is somewhat counterintuitive since the dimensionality effects are expected to raise the $T_{\text {MIT }}$ continuously by promoting the insulating state. ${ }^{17}$ We suggest the delicate changes of OOR in the SLs to be responsible for this anomaly. As $n$ is reduced below a critical thickness ( $\sim 6 \mathrm{uc})$, the OOR of the SLs changes to $a^{0} a^{0} c^{-}$by quenching the in-plane OOR given the limited coherence length of the interfacial OOR coupling., ${ }^{3,21,40}$ The quenched in-plane octahedral tilting can suppress the breathing mode instabilities in NNO and then weaken the MIT. ${ }^{29}$ Upon decreasing $n$ below 5 uc, the OOR of the SLs should be virtually unchanged, and the dominated dimensionality effects dramatically increase the $T_{\mathrm{MIT}}$ (Figure $3 \mathrm{c}$ ). To gain further insights into the thickness-dependent MIT, we fit the metallic resistivity of the SLs with $n \geq 3$ using the power law $\rho$ $=\rho_{0}+A T^{\alpha}$, where the parameter $A$ is determined by the strength of the electron scattering. ${ }^{44}$ The power-law exponent $\alpha$ is found to increase from $\alpha=1.0 \pm 0.1$ for $n \geq 5$ to $\alpha=1.90$ \pm 0.01 for $n=4$ and end up with $\alpha=3.14 \pm 0.01$ for $n=3$
(Figure S5). The departure from a linear scaling in perovskite nickelates has been ascribed to a joint effect of epitaxial strain and oxygen vacancies. ${ }^{44}$ Considering the fact that all SLs are coherently strained to the NGO substrate, the larger $\alpha$ for $n=$ 3,4 is probably due to the increasing amounts of oxygen vacancies with ultrathin NNO layers. This can also contribute to the enhanced resistivity and $T_{\mathrm{MIT}}$ by creating local disproportionation of $\mathrm{NiO}_{6}$ octahedra. ${ }^{45}$ The $n=2 \mathrm{SL}$ exhibits insulating behavior in the entire temperature range measured. The temperature dependent resistivity curve can be well fitted with the two-dimensional variable range hopping conduction model (Figure S6), attesting to the dimensional crossover. ${ }^{35}$ Note that the effect of disorder should not play a dominant role in the low-temperature insulating states for the SLs with $n$ $>2$. Otherwise we would have seen a monotonic increase of $\rho(10 \mathrm{~K})$ with reducing $n$.

The altered OOR should also have a profound impact on the antiferromagnetic transitions of the SLs. Here, the peak position of the $\mathrm{d}(\ln \rho) /[\mathrm{d} \ln (1 / T)]$ vs $T$ plot has been used to track $T_{\mathrm{N}}$ in $\mathrm{RNiO}_{3}{ }^{2,46}$ As shown in Figure $3 \mathrm{e}, T_{\mathrm{N}}$ nearly coincides with $T_{\text {MIT }}$ for the NNO single film, while they are well separated for all SLs. The decoupling of MIT and spin ordering has also been reported for other $\mathrm{RNiO}_{3}$-based heterostructures and SLs with different boundary conditions. ${ }^{2,9,47,48}$ The suppressed OOR along in-plane axes leads to a decrease of $T_{\mathrm{N}}$ from $\sim 168 \mathrm{~K}$ for the 25 -uc NNO film to $\sim 82 \mathrm{~K}$ for the $n=8 \mathrm{SL}$, following the trend in the bulk $\mathrm{RNiO}_{3}$ phase diagram. ${ }^{22}$ As $n$ decreases from 8 to $6 \mathrm{uc}, T_{\mathrm{N}}$ hardly changes due to the slightly altered OOR in this thickness range. Remarkably, the further blocking of the in-plane OOR reduces $T_{\mathrm{N}}$ from $\sim 91 \mathrm{~K}$ to $\sim 64 \mathrm{~K}$ as $n$ decreases from 6 to 5 uc. This drop of $T_{\mathrm{N}}$ is not substantial given the relatively small change of the absolute $\mathrm{Ni}-\mathrm{O}-\mathrm{Ni}$ bond angle. It is interesting to note that $T_{\mathrm{N}}$ remains nearly constant for $n \leq 5$ in contrast to a rapidly increasing $T_{\text {MIT }}$ (Figure $3 c$ ). For the insulating SL with $n=2$, the antiferromagnetic transition is manifested as an anomaly around $\sim 68 \mathrm{~K}$ of the $\mathrm{d}(\ln \rho) /[\mathrm{d} \ln (1 / T)]$ vs $T$ curve, corresponding to a kink visualized from the enlarged resistivity curve (Figure S7). This resembles $\mathrm{RNiO}_{3}$ with small rare-earth ions. ${ }^{46} \mathrm{X}$-ray resonant magnetic diffraction (RMD) measurements were performed for the SLs with $n=4$ and 2 (Figure 3d and Figure S7). The observation of the specific $q=(1 / 4,1 / 4$, $1 / 4)_{p c}$ reflection ascertains the presence of antiferromagnetic order in the SLs even with 2-uc NNO. The integrated peak intensity linearly decreases with increasing temperature, different from NNO single films which follow the mean field model. ${ }^{2,48}$ Such abnormal temperature dependence is in line with that reported for short-period $\mathrm{NNO} / \mathrm{NdAlO}_{3} \mathrm{SLs}$, which has been ascribed to the enhanced spin fluctuations in twodimensional systems. ${ }^{48}$ The linear extrapolations give onset temperatures of $\sim 93 \mathrm{~K}$ for $n=2$ and $\sim 90 \mathrm{~K}$ for $n=4 \mathrm{SL}$. The values are $\sim 30 \mathrm{~K}$ higher than those derived from resistivity measurements. Such a discrepancy arises because the RMD and derivative of resistivity characterize different stages of the antiferromagnetic transition. While RMD probes the onset of antiferromagnetic domains, the deflection point from the $\mathrm{d}(\mathrm{ln}$ $\rho) /[\mathrm{d} \ln (1 / T)]$ plot marks the percolation threshold. For twodimensional systems, the percolation occurs when the volume fraction of the antiferromagnetic insulating phase reaches $50 \%{ }^{49}$ Importantly, the RMD results strengthen the conclusion that $T_{\mathrm{N}}$ in SLs with $n \leq 5$ is stable against further reducing $n$ down to 2 uc. 

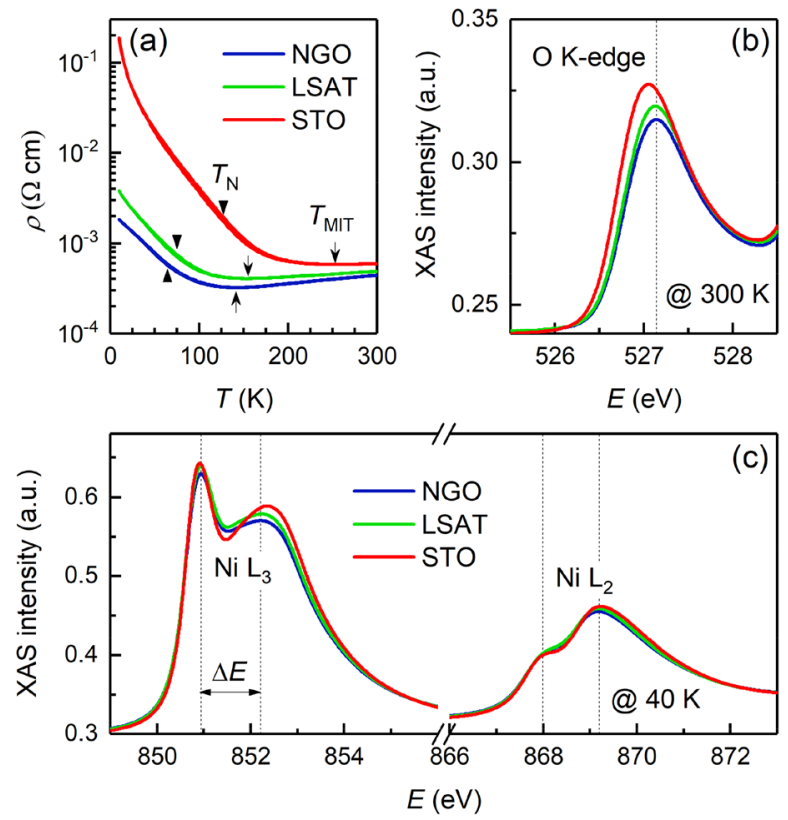
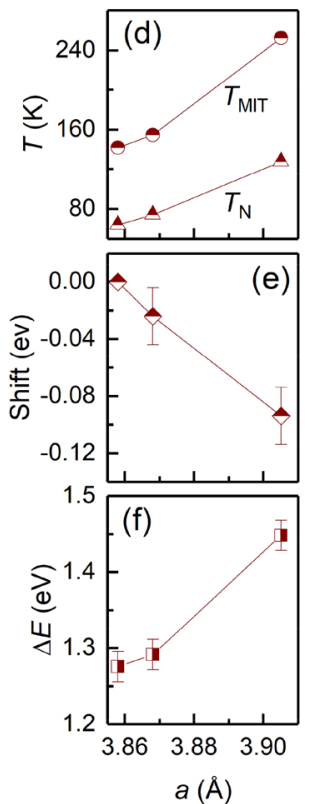

Figure 4. MITs in NNO/STO SLs with varied tetragonal distortions. (a) $\rho-T$ curves of the $n=5$ SLs grown on various substrates. $T_{\text {MIT }}$ and $T_{\mathrm{N}}$ are marked by arrows and triangles, respectively. (b) XAS spectra of the prepeak of the O K-edge at $300 \mathrm{~K}$. (c) XAS spectra of the Ni L-edge at 40 $\mathrm{K} . \Delta E$ is the splitting energy of the double peaks of the $\mathrm{Ni}_{3}$ edge. $(\mathrm{d}-\mathrm{f})$ Summary of the $T_{\mathrm{MIT}}, T_{\mathrm{N}}$, and energy shift of the $\mathrm{O} \mathrm{K}$-edge prepeak relative to the SL grown on NGO, as well as $\Delta E$ as a function of the in-plane lattice constant, $a$.

Taken together, the results suggest that $T_{\mathrm{N}}$ is mainly governed by the $\mathrm{Ni}-\mathrm{O}-\mathrm{Ni}$ bond angle and insensitive to the dimensional crossover in NNO/STO SLs. The argument is supported by the report on $\mathrm{NNO} / \mathrm{NdAlO}_{3} \mathrm{SLs}$, where a thickness-independent $T_{\mathrm{N}}$ of $\sim 150 \mathrm{~K}$ is observed even with a 2-uc thick NNO layer. ${ }^{48}$ The quasi-two-dimensional antiferromagnetic order preserved in $\mathrm{RNiO}_{3}$ is also consistent with previous observations in $\mathrm{LaNiO}_{3} / \mathrm{LaAlO}_{3}$ SLs, where an emergent antiferromagnetic order was probed in the otherwise paramagnetic $\mathrm{LaNiO}_{3}$ when the layer thickness was reduced to 2 uc. $^{13}$

To address the effects of varied tetragonal distortions on the MITs, we compared the MITs of NNO/STO SLs with $n=5$ grown on $(001)_{\mathrm{pc}}-\mathrm{NGO}\left(a_{\mathrm{pc}}=3.858 \AA\right),(001)-(\mathrm{LaA}-$ $\left.\mathrm{lO}_{3}\right)_{0.3}\left(\mathrm{Sr}_{2} \mathrm{AlTaO}_{6}\right)_{0.7}$ (LSAT, $a=3.868 \AA$ ), and (001)-STO $(a=3.905 \AA)$ substrates. RSM measurements confirm their fully strained states and identical tetragonal symmetry (Figure S8). The SLs grown on NGO and LSAT show very similar behavior, although the one on LSAT shows slightly higher $T_{\mathrm{MIT}}$ and $T_{\mathrm{N}}$ (Figure $4 \mathrm{a}, \mathrm{d}$ ). By contrast, the greater tetragonal distortion imposed by the STO substrate gives rise to substantially increased $T_{\mathrm{MIT}}$ and $T_{\mathrm{N}}$, as well as an enhanced resistivity jump across the MIT. To understand the varied MIT behaviors, the electronic structure was studied using XAS performed at $\mathrm{O} \mathrm{K}$ - and Ni L-edges. Figure $4 \mathrm{~b}$ shows the $\mathrm{O} \mathrm{K}$ edge prepeak which arises from the strong covalency between the Ni $3 \mathrm{~d}$ and $\mathrm{O} 2 \mathrm{p}$ states. The peak position is determined by the charge transfer energy. ${ }^{23,48,50}$ By taking the SL grown on NGO as a reference, the prepeak shifts $\sim 25 \mathrm{meV}$ and $\sim 95$ $\mathrm{meV}$ to lower energies for the SLs grown on LSAT and STO, respectively (Figure 4e). Such a red shift of the O K-edge prepeak can be assigned to an increasing charge transfer energy when the SL is subjected to a larger in-plane tensile strain., ${ }^{2,51}$ Figure $4 \mathrm{c}$ shows XAS at the $\mathrm{Ni}$ L-edge taken at $40 \mathrm{~K}$, well below $T_{\text {MIT }}$. Both the $\mathrm{Ni}_{2}$ - and $\mathrm{L}_{3}$-edges split into two peaks, resembling bulk $\mathrm{RNiO}_{3} \cdot{ }^{50,52}$ We define $\Delta E$ as the energy separation of the two $\mathrm{Ni} \mathrm{L}_{3}$ peaks given the well-defined double-peak feature. $\Delta E$ increases from $1.27 \mathrm{eV}$ on NGO to $1.29 \mathrm{eV}$ on LSAT and then to $1.45 \mathrm{eV}$ on STO (Figure 4f). The trend is in line with that of bulk $\mathrm{RNiO}_{3}$ upon reducing the ion radius of $R$, indicating that the charge transfer energy and breathing distortions of $\mathrm{NiO}_{6}$ octahedra increase with $a^{50,52}$ Our results suggest that robust MITs can be triggered by imposing a large tetragonal distortion to NNO/STO SLs, albeit with strongly suppressed OOR.

To summarize, the OOR of NNO/STO SLs evolves from a modulated to uniform pattern as we reduce the layer thickness of NNO. The altered OOR profoundly influences the MIT and the antiferromagnetic transition. We observe a reduced $T_{\text {MIT }}$ and $T_{N}$, together with a weakened insulating ground state as the OOR is suppressed. The dimensionality effect also plays a role in the MIT which raises $T_{\text {MIT }}$ by reducing the effective bandwidth. However, $T_{\mathrm{N}}$ is closely linked to the modifications of the crystal structure and is insusceptible to the dimensional crossover. Our results illustrate that the delicate interplay between the spatial confinement and the interfacial OOR coupling can be utilized to engineer novel electronic phases at the atomic level.

\section{ASSOCIATED CONTENT}

\section{sI Supporting Information}

The Supporting Information is available free of charge at https://pubs.acs.org/doi/10.1021/acs.nanolett.0c03850.

Materials and Methods section and supplementary Figures S1-S8 including RHEED intensity profile and patterns, AFM images, RSMs, STEM images and EELS mappings, fittings of the metallic resistivity, enlarged view of the $\rho-T$ curve, and RMD measurements (PDF) 


\section{AUTHOR INFORMATION}

\section{Corresponding Author}

Gertjan Koster - MESA+ Institute for Nanotechnology, University of Twente, 7500 AE Enschede, The Netherlands; (1) orcid.org/0000-0001-5478-7329; Email: g.koster@ utwente.nl

\section{Authors}

Binbin Chen - MESA+ Institute for Nanotechnology, University of Twente, 7500 AE Enschede, The Netherlands

Nicolas Gauquelin - Electron Microscopy for Materials Science (EMAT), University of Antwerp, 2020 Antwerp, Belgium

Robert J. Green - Department of Physics and Engineering Physics, University of Saskatchewan, Saskatoon, Saskatchewan S7N 5E2, Canada; Stewart Blusson Quantum Matter Institute, University of British Columbia, Vancouver, British Columbia V6T 1Z4, Canada

Jin Hong Lee - Unité Mixte de Physique, CNRS, Thales, Univ. Paris-Sud, Université Paris-Saclay, 91767 Palaiseau, France; 이이.orcido000-0002-1423-1438

Cinthia Piamonteze - Swiss Light Source, Paul Scherrer Institute, 5232 Villigen, Switzerland; (1) orcid.org/00000002-8416-9668

Matjaž Spreitzer - Advanced Materials Department, Jožef Stefan Institute, 1000 Ljubljana, Slovenia

Daen Jannis - Electron Microscopy for Materials Science (EMAT), University of Antwerp, 2020 Antwerp, Belgium

Johan Verbeeck - Electron Microscopy for Materials Science (EMAT), University of Antwerp, 2020 Antwerp, Belgium

Manuel Bibes - Unité Mixte de Physique, CNRS, Thales, Univ. Paris-Sud, Université Paris-Saclay, 91767 Palaiseau, France; (1) orcid.org/0000-0002-6704-3422

Mark Huijben - MESA+ Institute for Nanotechnology, University of Twente, 7500 AE Enschede, The Netherlands; (1) orcid.org/0000-0001-8175-6958

Guus Rijnders - MESA+ Institute for Nanotechnology, University of Twente, 7500 AE Enschede, The Netherlands

Complete contact information is available at:

https://pubs.acs.org/10.1021/acs.nanolett.0c03850

\section{Author Contributions}

B.C. synthesized the samples and conducted the basic structural characterizations and transport measurements. N.G. and D.J. performed the STEM measurements and analyses under the supervision of J.V. R.J.G. performed the RMD measurements. B.C. performed the XAS measurements assisted by J.H.L. and C.P. B.C., N.G., M.S., M.B., M.H., G.R., and G.K. discussed the results. B.C. and G.K. conceived the project and wrote the manuscript with inputs from all authors.

\section{Notes}

The authors declare no competing financial interest.

\section{ACKNOWLEDGMENTS}

This work is supported by the international M-ERA.NET project SIOX (project 4288). J.V. and N.G. acknowledge funding through the GOA project "Solarpaint" of the University of Antwerp. The microscope used in this work was partly funded by the Hercules Fund from the Flemish Government. D.J. acknowledges funding from FWO Project G093417N from the Flemish fund for scientific research. M.S. acknowledges funding from Slovenian Research Agency
(Grants J2-9237 and P2-0091). R.J.G. acknowledges funding from the Natural Sciences and Engineering Research Council of Canada (NSERC). Part of the research described in this paper was performed at the Canadian Light Source, a national research facility of the University of Saskatchewan, which is supported by the Canada Foundation for Innovation (CFI), NSERC, the National Research Council (NRC), the Canadian Institutes of Health Research (CIHR), the Government of Saskatchewan, and the University of Saskatchewan. This work received support from the ERC CoG MINT (No. 615759) and from a PHC Van Gogh grant. M.B. thanks the French Academy of Science and the Royal Netherlands Academy of Arts and Sciences for supporting his stays in The Netherlands. This project has received funding from the European Union's Horizon 2020 research and innovation programme under grant agreement No. 823717 - ESTEEM3.

\section{REFERENCES}

(1) Schlom, D. G.; Chen, L.-Q.; Fennie, C. J.; Gopalan, V.; Muller, D. A.; Pan, X.; Ramesh, R.; Uecker, R. Elastic Strain Engineering of Ferroic Oxides. MRS Bull. 2014, 39, 118-130.

(2) Liu, J.; Kargarian, M.; Kareev, M.; Gray, B.; Ryan, P. J.; Cruz, A.; Tahir, N.; Chuang, Y.-D.; Guo, J.; Rondinelli, J. M.; Freeland, J. W.; Fiete, G. A.; Chakhalian, J. Heterointerface Engineered Electronic and Magnetic Phases of $\mathrm{NdNiO}_{3}$ Thin Films. Nat. Commun. 2013, 4 2714.

(3) Rondinelli, J. M.; May, S. J.; Freeland, J. W. Control of Octahedral Connectivity in Perovskite Oxide Heterostructures: An Emerging Route to Multifunctional Materials Discovery. MRS Bull. 2012, 37, 261-270.

(4) Rondinelli, J. M.; Spaldin, N. A. Structure and Properties of Functional Oxide Thin Films: Insights from Electronic-Structure Calculations. Adv. Mater. 2011, 23, 3363-3381.

(5) Liao, Z.; Huijben, M.; Zhong, Z.; Gauquelin, N.; Macke, S.; Green, R. J.; Van Aert, S.; Verbeeck, J.; Van Tendeloo, G.; Held, K.; Sawatzky, G. A.; Koster, G.; Rijnders, G. Controlled Lateral Anisotropy in Correlated Manganite Heterostructures by InterfaceEngineered Oxygen Octahedral Coupling. Nat. Mater. 2016, 15, 425431.

(6) Kan, D.; Aso, R.; Sato, R.; Haruta, M.; Kurata, H.; Shimakawa, Y. Tuning Magnetic Anisotropy by Interfacially Engineering the Oxygen Coordination Environment in a Transition Metal Oxide. Nat. Mater. 2016, 15, 432-437.

(7) Moon, E. J.; Colby, R.; Wang, Q.; Karapetrova, E.; Schlepütz, C. M.; Fitzsimmons, M. R.; May, S. J. Spatial Control of Functional Properties via Octahedral Modulations in Complex Oxide Superlattices. Nat. Commun. 2014, 5, 5710.

(8) Moon, E. J.; Balachandran, P. V.; Kirby, B. J.; Keavney, D. J.; Sichel-Tissot, R. J.; Schlepütz, C. M.; Karapetrova, E.; Cheng, X. M.; Rondinelli, J. M.; May, S. J. Effect of Interfacial Octahedral Behavior in Ultrathin Manganite Films. Nano Lett. 2014, 14, 2509-2514.

(9) Liao, Z.; Gauquelin, N.; Green, R. J.; Müller-Caspary, K.; Lobato, I.; Li, L.; Van Aert, S.; Verbeeck, J.; Huijben, M.; Grisolia, M. N.; Rouco, V.; El Hage, R.; Villegas, J. E.; Mercy, A.; Bibes, M.; Ghosez, P.; Sawatzky, G. A.; Rijnders, G.; Koster, G. Metal-InsulatorTransition Engineering by Modulation Tilt-Control in Perovskite Nickelates for Room Temperature Optical Switching. Proc. Natl. Acad. Sci. U. S. A. 2018, 115, 9515-9520.

(10) Liao, Z.; Green, R. J.; Gauquelin, N.; Macke, S.; Li, L.; Gonnissen, J.; Sutarto, R.; Houwman, E. P.; Zhong, Z.; Van Aert, S.; Verbeeck, J.; Sawatzky, G. A.; Huijben, M.; Koster, G.; Rijnders, G. Long-Range Domain Structure and Symmetry Engineering by Interfacial Oxygen Octahedral Coupling at Heterostructure Interface. Adv. Funct. Mater. 2016, 26, 6627-6634.

(11) Bousquet, E.; Dawber, M.; Stucki, N.; Lichtensteiger, C.; Hermet, P.; Gariglio, S.; Triscone, J.-M.; Ghosez, P. Improper 
Ferroelectricity in Perovskite Oxide Artificial Superlattices. Nature 2008, 452, 732-736.

(12) May, S. J.; Smith, C. R.; Kim, J. W.; Karapetrova, E.; Bhattacharya, A.; Ryan, P. J. Control of Octahedral Rotations in $\left(\mathrm{LaNiO}_{3}\right)_{\mathrm{n}} /\left(\mathrm{SrMnO}_{3}\right)_{\mathrm{m}}$ Superlattices. Phys. Rev. B: Condens. Matter Mater. Phys. 2011, 83, 153411.

(13) Boris, A. V.; Matiks, Y.; Benckiser, E.; Frano, A.; Popovich, P.; Hinkov, V.; Wochner, P.; Castro-Colin, M.; Detemple, E.; Malik, V. K.; Bernhard, C.; Prokscha, T.; Suter, A.; Salman, Z.; Morenzoni, E.; Cristiani, G.; Habermeier, H.-U.; Keimer, B. Dimensionality Control of Electronic Phase Transitions in Nickel-Oxide Superlattices. Science 2011, 332, 937-940.

(14) Sakai, E.; Tamamitsu, M.; Yoshimatsu, K.; Okamoto, S.; Horiba, K.; Oshima, M.; Kumigashira, H. Gradual Localization of $\mathrm{Ni}$ 3d States in $\mathrm{LaNiO}_{3}$ Ultrathin Films Induced by Dimensional Crossover. Phys. Rev. B: Condens. Matter Mater. Phys. 2013, 87, 075132.

(15) King, P. D. C.; Wei, H. I.; Nie, Y. F.; Uchida, M.; Adamo, C.; Zhu, S.; He, X.; Božović, I.; Schlom, D. G.; Shen, K. M. Atomic-Scale Control of Competing Electronic Phases in Ultrathin $\mathrm{LaNiO}_{3}$. Nat. Nanotechnol. 2014, 9, 443-447.

(16) Sakai, E.; Yoshimatsu, K.; Tamamitsu, M.; Horiba, K.; Fujimori, A.; Oshima, M.; Kumigashira, H. Bandwidth-Controlled MetalInsulator Transition in Epitaxial $\mathrm{PrNiO}_{3}$ Ultrathin Films Induced by Dimensional Crossover. Appl. Phys. Lett. 2014, 104, 171609.

(17) Wang, L.; Ju, S.; You, L.; Qi, Y.; Guo, Y. W.; Ren, P.; Zhou, Y.; Wang, J. Competition between Strain and Dimensionality Effects on the Electronic Phase Transitions in $\mathrm{NdNiO}_{3}$ Films. Sci. Rep. 2016, 5, 18707.

(18) Chen, P. F.; Chen, B. B.; Tan, X. L.; Xu, H. R.; Xuan, X. F.; Guo, Z.; Jin, F.; Wu, W. B. High- $\mathrm{T}_{\mathrm{C}}$ Ferromagnetic Order in $\mathrm{CaRuO}_{3} / \mathrm{La}_{2 / 3} \mathrm{Ca}_{1 / 3} \mathrm{MnO}_{3}$ Superlattices. Appl. Phys. Lett. 2013, 103, 262402.

(19) Chen, B.; Chen, P.; Xu, H.; Jin, F.; Guo, Z.; Lan, D.; Wan, S.; Gao, G.; Chen, F.; Wu, W. Interfacial Control of Ferromagnetism in Ultrathin $\mathrm{La}_{0.67} \mathrm{Ca}_{0.33} \mathrm{MnO}_{3}$ Sandwiched between $\mathrm{CaRu}_{1-x} \mathrm{Ti}_{x} \mathrm{O}_{3}(x=$ 0-0.8) Epilayers. ACS Appl. Mater. Interfaces 2016, 8, 34924-34932.

(20) Qiao, L.; Jang, J. H.; Singh, D. J.; Gai, Z.; Xiao, H.; Mehta, A.; Vasudevan, R. K.; Tselev, A.; Feng, Z.; Zhou, H.; Li, S.; Prellier, W.; Zu, X.; Liu, Z.; Borisevich, A.; Baddorf, A. P.; Biegalski, M. D. Dimensionality Controlled Octahedral Symmetry-Mismatch and Functionalities in Epitaxial $\mathrm{LaCoO}_{3} / \mathrm{SrTiO}_{3}$ Heterostructures. Nano Lett. 2015, 15, 4677-4684.

(21) Schütz, P.; Di Sante, D.; Dudy, L.; Gabel, J.; Stübinger, M.; Kamp, M.; Huang, Y.; Capone, M.; Husanu, M.-A.; Strocov, V. N.; Sangiovanni, G.; Sing, M.; Classen, R. Dimensionality-Driven MetalInsulator Transition in Spin-Orbit-Coupled $\mathrm{SrIrO}_{3}$. Phys. Rev. Lett. 2017, 119, 256404.

(22) Catalan, G. Progress in Perovskite Nickelate Research. Phase Transitions 2008, 81, 729-749.

(23) Middey, S.; Chakhalian, J.; Mahadevan, P.; Freeland, J. W.; Millis, A. J.; Sarma, D. D. Physics of Ultrathin Films and Heterostructures of Rare Earth Nickelates. Annu. Rev. Mater. Res. 2016, 46, 305-334.

(24) Alonso, J. A.; García-Muñoz, J. L.; Fernández-Díaz, M. T.; Aranda, M. A. G.; Martínez-Lope, M. J.; Casais, M. T. Charge Disproportionation in $\mathrm{RNiO}_{3}$ Perovskites: Simultaneous MetalInsulator and Structural Transition in $\mathrm{YNiO}_{3}$. Phys. Rev. Lett. 1999, 82, 3871-3874.

(25) Mizokawa, T.; Khomskii, D. I.; Sawatzky, G. A. Spin and Charge Ordering in Self-Doped Mott Insulators. Phys. Rev. B: Condens. Matter Mater. Phys. 2000, 61, 11263.

(26) Stewart, M. K.; Liu, J.; Kareev, M.; Chakhalian, J.; Basov, D. N. Mott Physics near the Insulator-to-Metal Transition in $\mathrm{NdNiO}_{3}$. Phys. Rev. Lett. 2011, 107, 176401.

(27) Johnston, S.; Mukherjee, A.; Elfimov, I.; Berciu, M.; Sawatzky, G. A. Charge Disproportionation without Charge Transfer in the Rare-Earth-Element Nickelates as a Possible Mechanism for the Metal-Insulator Transition. Phys. Rev. Lett. 2014, 112, 106404.
(28) Upton, M. H.; Choi, Y.; Park, H.; Liu, J.; Meyers, D.; Chakhalian, J.; Middey, S.; Kim, J.-W.; Ryan, P. J. Novel electronic behavior driving $\mathrm{NdNiO}_{3}$ metal-insulator transition. Phys. Rev. Lett. 2015, 115, 036401.

(29) Mercy, A.; Bieder, J.; Íniguez, J.; Ghosez, P. Structurally triggered metal-insulator transition in rare-earth nickelates. Nat. Commun. 2017, 8, 1677.

(30) Varignon, J.; Grisolia, M. N.; Íñiguez, J.; Barthélémy, A.; Bibes, M. Complete phase diagram of rare-earth nickelates from firstprinciples. npj Quantum Mater. 2017, 2, 21.

(31) Bisogni, V.; Catalano, S.; Green, R. J.; Gibert, M.; Scherwitzl, R.; Huang, Y.; Strocov, V. N.; Zubko, P.; Balandeh, S.; Triscone, J.M.; Sawatzky, G.; Schmitt, T. Ground-state oxygen holes and the metal-insulator transition in the negative charge-transfer rare-earth nickelates. Nat. Commun. 2016, 7, 13017.

(32) Chaloupka, J.; Khaliullin, G. Orbital Order and Possible Superconductivity in $\mathrm{LaNiO}_{3} / \mathrm{LaMO}_{3}$ Superlattices. Phys. Rev. Lett. 2008, 100, 016404.

(33) Hansmann, P.; Yang, X.; Toschi, A.; Khaliullin, G.; Andersen, O. K.; Held, K. Turning a nickelate Fermi surface into a cupratelike one through heterostructuring. Phys. Rev. Lett. 2009, 103, 016401.

(34) Liu, J.; Kareev, M.; Meyers, D.; Gray, B.; Ryan, P.; Freeland, J. W.; Chakhalian, J. Metal-Insulator Transition and Orbital Reconstruction in Mott-Type Quantum Wells Made of $\mathrm{NdNiO}_{3}$. Phys. Rev. Lett. 2012, 109, 107402.

(35) Scherwitzl, R.; Gariglio, S.; Gabay, M.; Zubko, P.; Gibert, M.; Triscone, J.-M. Metal-insulator transition in ultrathin $\mathrm{LaNiO}_{3}$ films. Phys. Rev. Lett. 2011, 106, 246403.

(36) Miao, N.; Bristowe, N. C.; Xu, B.; Verstraete, M. J.; Ghosez, P. First-Principles Study of the Lattice Dynamical Properties of Strontium Ruthenate. J. Phys.: Condens. Matter 2014, 26, 035401.

(37) Zhang, J. Y.; Hwang, J.; Raghavan, S.; Stemmer, S. Symmetry lowering in extreme-electron-density perovskite quantum wells. Phys. Rev. Lett. 2013, 110, 256401.

(38) Chang, S. H.; Chang, Y. J.; Jang, S. Y.; Jeong, D. W.; Jung, C. U.; Kim, Y. J.; Chung, J. S.; Noh, T. W. Thickness-Dependent Structural Phase Transition of Strained $\mathrm{SrRuO}_{3}$ Ultrathin Films: The Role of Octahedral Tilt. Phys. Rev. B: Condens. Matter Mater. Phys. 2011, 84, 104101.

(39) Kan, D.; Wakabayashi, Y.; Tajiri, H.; Shimakawa, Y. Interfacially engineered oxygen octahedral rotations and their impact on strain relief in coherently grown $\mathrm{SrRuO}_{3}$ films. Phys. Rev. B: Condens. Matter Mater. Phys. 2016, 94, 024112.

(40) Vailionis, A.; Boschker, H.; Liao, Z.; Smit, J. R. A.; Rijnders, G.; Huijben, M.; Koster, G. Symmetry and lattice mismatch induced strain accommodation near and away from correlated perovskite interfaces. Appl. Phys. Lett. 2014, 105, 131906.

(41) Hwang, J.; Son, J.; Zhang, J. Y.; Janotti, A.; Van De Walle, C. G.; Stemmer, S. Structural Origins of the Properties of Rare Earth Nickelate Superlattices. Phys. Rev. B: Condens. Matter Mater. Phys. 2013, 87, 060101.

(42) Xiang, P.-H.; Zhong, N.; Duan, C.-G.; Tang, X. D.; Hu, Z. G.; Yang, P. X.; Zhu, Z. Q.; Chu, J. H. Strain Controlled Metal-Insulator Transition in Epitaxial $\mathrm{NdNiO}_{3}$ Thin Films. J. Appl. Phys. 2013, 114, 243713.

(43) Breckenfeld, E.; Chen, Z.; Damodaran, A. R.; Martin, L. W. Effects of Nonequilibrium Growth, Nonstoichiometry, and Film Orientation on the Metal-to-Insulator Transition in $\mathrm{NdNiO}_{3}$ Thin Films. ACS Appl. Mater. Interfaces 2014, 6, 22436-22444.

(44) Guo, Q.; Farokhipoor, S.; Magén, C.; Rivadulla, F.; Noheda, B. Tunable Resistivity Exponents in the Metallic Phase of Epitaxial Nickelates. Nat. Commun. 2020, 11, 2949.

(45) Kim, T. H.; Paudel, T. R.; Green, R. J.; Song, K.; Lee, H. S.; Choi, S. Y.; Irwin, J.; Noesges, B.; Brillson, L. J.; Rzchowski, M. S.; Sawatzky, G. A.; Tsymbal, E. Y.; Eom, C. B. Strain-Driven Disproportionation at a Correlated Oxide Metal-Insulator Transition. Phys. Rev. B: Condens. Matter Mater. Phys. 2020, 101, 121105. 
(46) Zhou, J. S.; Goodenough, J. B.; Dabrowski, B. Exchange Interaction in the Insulating Phase of $\mathrm{RNiO}_{3}$. Phys. Rev. Lett. 2005, 95, 127204.

(47) Middey, S.; Meyers, D.; Kareev, M.; Cao, Y.; Liu, X.; Shafer, P.; Freeland, J. W.; Kim, J.-W.; Ryan, P. J.; Chakhalian, J. Disentangled Cooperative Orderings in Artificial Rare-Earth Nickelates. Phys. Rev. Lett. 2018, 120, 156801.

(48) Disa, A. S.; Georgescu, A. B.; Hart, J. L.; Kumah, D. P.; Shafer, P.; Arenholz, E.; Arena, D. A.; Ismail-Beigi, S.; Taheri, M. L.; Walker, F. J.; Ahn, C. H. Control of hidden ground-state order in $\mathrm{NdNiO}_{3}$ superlattices. Phys. Rev. Mater. 2017, 1, 024410.

(49) Catalan, G.; Bowman, R. M.; Gregg, J. M. Metal-insulator transitions in $\mathrm{NdNiO}_{3}$ thin films. Phys. Rev. B: Condens. Matter Mater. Phys. 2000, 62, 7892.

(50) Freeland, J. W.; Van Veenendaal, M.; Chakhalian, J. Evolution of Electronic Structure across the Rare-Earth $\mathrm{RNiO}_{3}$ Series. J. Electron Spectrosc. Relat. Phenom. 2016, 208, 56-62.

(51) Chakhalian, J.; Rondinelli, J. M.; Liu, J.; Gray, B. A.; Kareev, M.; Moon, E. J.; Prasai, N.; Cohn, J. L.; Varela, M.; Tung, I. C.; Bedzyk, M. J.; Altendorf, S. G.; Strigari, F.; Dabrowski, B.; Tjeng, L. H.; Ryan, P. J.; Freeland, J. W. Asymmetric Orbital-Lattice Interactions in Ultrathin Correlated Oxide Films. Phys. Rev. Lett. 2011, 107, 116805.

(52) Piamonteze, C.; de Groot, F. M. F.; Tolentino, H. C. N.; Ramos, A. Y.; Massa, N. E.; Alonso, J. A.; Martínez-Lope, M. J. Spinorbit-induced mixed-spin ground state in $\mathrm{RNiO}_{3}$ perovskites probed by $\mathrm{x}$-ray absorption spectroscopy: Insight into the metal-to-insulator transition. Phys. Rev. B: Condens. Matter Mater. Phys. 2005, 71, 020406. 